International Journal of Pure and Applied Mathematics

Volume 115 No. 4 2017, 693-712

ISSN: 1311-8080 (printed version); ISSN: 1314-3395 (on-line version)

url: http://www.ijpam.eu

doi: $10.12732 /$ ijpam.v115i4.4

ijpam.eu

\title{
WEAKLY $\alpha$-CONTINUOUS FUZZY MULTIFUNCTIONS
}

\author{
Anjana Bhattacharyya \\ Department of Mathematics \\ Victoria Institution (College) \\ 78 B, A.P.C. Road, Kolkata, 700009, INDIA
}

\begin{abstract}
In this paper we have defined three new types of fuzzy multifunctions and several characterizations are done. Also a mutual relationship between these fuzzy multifunctions are established. In the last section some applications of these multifunctions are shown.
\end{abstract}

AMS Subject Classification: 54A40, 03E72

Key Words: fuzzy upper (lower) weakly $\alpha$-continuous multifunctions, fuzzy $\alpha$-preopen multifunction, fuzzy regular space, fuzzy almost compact space, fuzzy upper (lower) $C$-nbd

\section{Introduction}

Fuzzy multifunction, a function from an ordinary topological space $X$ into a fuzzy topological space $Y$ was introduced by Papageorgiou [19] in 1985 which has paved a new direction to many researchers to carry on their interest in this area. He also defined fuzzy upper and lower inverses and fuzzy upper and lower semi continuity. Afterwards, it was noticed in [14] that the definition of fuzzy lower inverse and fuzzy lower semi continuity were not natural so that Mukherjee and Malakar [14] redefined these two notions via q-coincidence and q-neighbourhoods of $\mathrm{Pu}$ and Liu [21]. In this paper, the definition of fuzzy upper inverse and fuzzy upper semi continuity of Papageorgiou [19] and fuzzy lower inverse and fuzzy lower semi continuity of Mukherjee and Malakar[14] have been used.

$\begin{array}{lr}\text { Received: } & \text { September 29, } 2016 \\ \text { Revised: } & \text { May 24, } 2017 \\ \text { Published: } & \text { August 2, } 2017\end{array}$

(C) 2017 Academic Publications, Ltd. url: www.acadpubl.eu 


\section{Preliminaries}

Throughout the paper, $(X, \tau)$ or simply $X$ will stand for an ordinary topological space, while by $\left(Y, \tau_{Y}\right)$ or simply by $Y$ will always mean a fuzzy topological space (fts, for short) in the sense of Chang [6]. A fuzzy set [23] $A$ is a mapping from a nonempty set $Y$ into the closed interval $I=[0,1]$, i.e., $A \in I^{Y}$. The support of a fuzzy set $A$ in $Y$ will be denoted as supp $A$ [23] and is defined by $\operatorname{supp} A=\{y \in Y: A(y) \neq 0\}$. A fuzzy point [21] with the singleton support $y \in Y$ and the value $\alpha(0<\alpha \leq 1)$ at $y$ will be denoted by $y_{\alpha} \cdot 0_{Y}$ and $1_{Y}$ are the constant fuzzy sets taking respectively the constant values 0 and 1 on $Y$. The complement of a fuzzy set $A$ in $Y$ will be denoted by $1_{Y} \backslash A[23]$ and is defined by $\left(1_{Y} \backslash A\right)(y)=1-A(y)$, for all $y \in Y$. For two fuzzy sets $A$ and $B$ in $Y$, we write $A \leq B$ iff $A(y) \leq B(y)$, for each $y \in Y$, while we write $A q B$ to mean $A$ is quasi-coincident (q-coincident, for short) with $B$ [21] if there exists $y \in Y$ such that $A(y)+B(y)>1$; the negation of $A q B$ is written as $A \not q B$. cl $A$ and int $A$ of a set $A$ in $X$ (respectively, a fuzzy set $A$ in $Y$ ) respectively stand for the closure and interior (resp., fuzzy closure and fuzzy interior [6]) of $A$ in $X$ (respectively, in $Y$ ).

A fuzzy set $A$ in $Y$ is called fuzzy regular closed [2] if $A=\operatorname{clint} A$. A (fuzzy) set $A$ in $X$ (in $Y$ ) is said to be semiopen (fuzzy semiopen, fuzzy $\alpha$ open) in $X$ (in $Y$ ) if $A \subseteq \operatorname{clint} A$ (resp., $A \leq \operatorname{clint} A[2], A \leq \operatorname{int} \operatorname{clint} A[5])$ [10]. The complement of a semiopen (fuzzy semiopen, fuzzy $\alpha$-open) set in $X$ (in $Y$ ) is called semiclosed (fuzzy semiclosed [2], fuzzy $\alpha$-closed [5]) [10] set in $X$ (in $Y$ ). The intersection of all (fuzzy) semiclosed (fuzzy $\alpha$-closed) sets in $X$ (in $Y$ ) containing a (fuzzy) set $A$ in $X$ (in $Y$ ) is called (fuzzy) semiclosure (fuzzy $\alpha$-closure [5]) [10] ([2]) of $A$ and is denoted by $\operatorname{scl} A(\alpha c l A)$. The set of all semiopen sets containing a fuzzy point $x$ in $X$ is denoted by $S O(X, x)$. A set $A$ in $X$ is called preopen (resp., $\alpha$-open) if $A \subseteq \operatorname{intcl} A$ (resp., $A \subseteq \operatorname{intclint} A[12]$ ) [11]. The set of all preopen sets in $X$ is denoted by $P O(X)$. The complement of an $\alpha$-open set in $X$ is called $\alpha$-closed set. The union of all $\alpha$-open sets contained in a set $A$ in $X$ is called $\alpha$-interior of $A$, to be denoted by $\alpha-\operatorname{int} A$ [12]. The intersection of all $\alpha$-closed sets containing a set $A$ in $X$ is called $\alpha$-closure of $A$, to be denoted by $\alpha c l A[12]$. A set $U$ in $X$ is said to be an $\alpha$-nbd of a point $x \in X$ if there exists an $\alpha$-open set $V$ in $X$ such that $x \in V \subseteq U$ [12]. The set of all $\alpha$-open sets containing a point $x \in X$ is denoted by $\alpha O(X, x)$. A fuzzy point $y_{t}$ is called a fuzzy $\theta$-cluster point of a fuzzy set $A$ in $Y$ if $c l U q A$ for every fuzzy open set $U$ in $Y$ with $y_{t} q U$ [16]. The union of all fuzzy $\theta$-cluster points of a fuzzy set $A$ is called fuzzy $\theta$-closure [16] of $A$, to be denoted by $\theta \operatorname{cl} A$. A fuzzy set $A$ in $Y$ is called fuzzy $\theta$-closed if $A=\theta \operatorname{cl} A$ [16]. The complement of a 
fuzzy $\theta$-closed set is called fuzzy $\theta$-open [16]. The union of all fuzzy $\theta$-open sets contained in a fuzzy set $A$ in $Y$ is called fuzzy $\theta$-interior of $A$, to be denoted by $\theta-i n t A[16]$. A fuzzy set $A$ in $Y$ is fuzzy $\theta$-open if $A=\theta-i n t A$ [16]. It is known that $\theta \operatorname{cl} A$ is fuzzy closed for any fuzzy set $A$ in $Y$ and $\operatorname{cl} A=\theta \operatorname{cl} A$ for any fuzzy open set $A$ in $Y[16]$.

\section{Some Well Known Definitions, Theorems and Lemmas}

Let us recall the following definition from [19] for ready references.

Definition 3.1 [19]. Let $(X, \tau)$ and $\left(Y, \tau_{Y}\right)$ be respectively an ordinary topological space and an fts. We say that $F: X \rightarrow Y$ is a fuzzy multifunction if corresponding to each $x \in X, F(x)$ is a unique fuzzy set in $Y$.

Henceforth by $F: X \rightarrow Y$ we shall mean a fuzzy multifunction in the above sense.

Definition $3.2[19,14]$. For a fuzzy multifunction $F: X \rightarrow Y$, the upper inverse $F^{+}$and lower inverse $F^{-}$are defined as follows:

For any fuzzy set $A$ in $Y, F^{+}(A)=\{x \in X: F(x) \leq A\}$ and $F^{-}(A)=$ $\{x \in X: F(x) q A\}$.

There is a following relationship between the upper and the lower inverses of a fuzzy multifunction.

Theorem 3.3 [14]. For a fuzzy multifunction $F: X \rightarrow Y$, we have $F^{-}\left(1_{Y} \backslash\right.$ $A)=X \backslash F^{+}(A)$, for any fuzzy set $A$ in $Y$.

Definition 3.4 [6]. Let $A$ be a fuzzy set in an fts $Y$. A collection $\mathcal{U}$ of fuzzy sets in $Y$ is said to be a fuzzy cover of $A$ if $\sup \{U(x): U \in \mathcal{U}\}=1$ for each $x \in \operatorname{supp} A$. If, in addition, each member of $\mathcal{U}$ is fuzzy open, then $\mathcal{U}$ is called a fuzzy open cover of $A$. In particular, if $A=1_{Y}$, we get the definition of fuzzy cover (fuzzy open cover) of the fts. $Y$.

Definition 3.5 [7]. A fuzzy cover $\mathcal{U}$ of a fuzzy set $A$ in an fts $Y$ is said to have a finite subcover $\mathcal{U}_{0}$ if $\mathcal{U}_{0}$ is a finite subcollection of $\mathcal{U}$ such that $\bigcup \mathcal{U}_{0} \geq A$. Clearly, if $A=1_{Y}$, in particular, then the requirements on $\mathcal{U}_{0}$ is $\cup \mathcal{U}_{0}=1_{Y}$.

Definition 3.6. An fts $Y$ is called fuzzy compact [6] (resp., fuzzy almost compact [15]) space if every fuzzy open cover $\mathcal{U}$ of $Y$ has a finite subcover (resp., finite proximate subcover, i.e., there exists a finite subcollection $\mathcal{U}_{0}$ of $\mathcal{U}$ such that $\left.\bigcup_{U \in \mathcal{U}_{0}} c l U=1_{Y}\right)$. 
Lemma $3.7[20]$. The following properties hold for a subset $A$ of a topological space $X$ :

(1) If $A$ is open in $X$, then $\operatorname{scl} A=\operatorname{intcl} A$.

(2) $A$ is $\alpha$-open in $X$ iff there is some open set $U$ in $X$ such that $U \subset A \subset$ $s c l U$.

(3) $\alpha \operatorname{cl} A=A \bigcup \operatorname{cl}(\operatorname{int}(\operatorname{cl} A))$.

Lemma $3.8[3]$. For a fuzzy set $A$ in an $\mathrm{fts}\left(Y, \tau_{Y}\right), \operatorname{scl} A=$ intcl $A$.

\section{Fuzzy Upper and Lower Weakly $\alpha$-Continuous Multifunctions}

In this section we first introduce three types of fuzzy multifunctions and characterized them in several ways.

Definition 4.1. A fuzzy multifunction $F: X \rightarrow Y$ is called fuzzy:

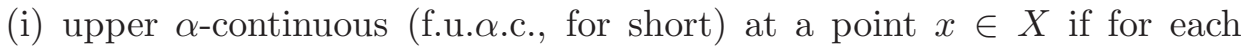
fuzzy open set $V$ of $Y$ containing $F(x)$, there exists $U \in \alpha O(X, x)$ such that $F(U) \leq V$,

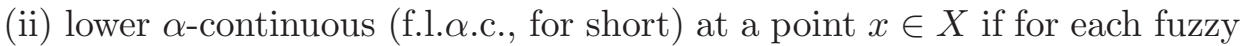
open set $V$ of $Y$ with $F(x) q V$, there exists $U \in \alpha O(X, x)$ such that $F(u) q V$, for all $u \in U$,

(iii) upper (lower) $\alpha$-continuous on $X$ if $F$ has this property at each point of $X$.

Definition 4.2. A fuzzy multifunction $F: X \rightarrow Y$ is said to be fuzzy:

(i) upper almost $\alpha$-continuous (f.u.a. $\alpha . c .$, for short) at a point $x \in X$ for each $U \in S O(X, x)$ and each fuzzy open set $V$ containing $F(x)$, there exists a non empty open set $G$ in $X$ with $G \subset U$ such that $F(G) \leq s c l V$,

(ii) lower almost $\alpha$-continuous (f.l.a. $\alpha . c$., for short) at a point $x \in X$ if for each $U \in S O(X, x)$ and each fuzzy open set $V$ with $F(x) q V$, there exists a non empty open set $G$ in $X$ with $G \subset U$ such that $F(g) q s c l V$, for all $g \in G$,

(iii) upper (lower) almost $\alpha$-continuous if it is fuzzy upper (lower) almost $\alpha$-continuous at every point of $X$.

Definition 4.3. A fuzzy multifunction $F: X \rightarrow Y$ is said to be fuzzy:

(i) upper weakly $\alpha$-continuous (f.u.w. $\alpha . c$. , for short) at a point $x \in X$ for each $U \in S O(X, x)$ and each fuzzy open set $V$ containing $F(x)$, there exists a non empty open set $G$ in $X$ with $G \subset U$ such that $F(G) \leq c l V$,

(ii) lower weakly $\alpha$-continuous (f.l.w. $\alpha . c$., for short) at a point $x \in X$ if for each $U \in S O(X, x)$ and each fuzzy open set $V$ with $F(x) q V$, there exists a non empty open set $G$ in $X$ with $G \subset U$ such that $F(g) q c l V$, for all $g \in G$, 
(iii) upper (lower) weakly $\alpha$-continuous if it is fuzzy upper (lower) weakly $\alpha$-continuous at every point of $X$.

Theorem 4.4. For a fuzzy multifunction $F: X \rightarrow Y$, the following statements are equivalent:

(i) $F$ is f.u.w. $\alpha . c$. at a point $x \in X$,

(ii) for any fuzzy open set $V$ of $Y$ with $F(x) \leq V$, there exists $S \in \alpha O(X, x)$ such that $F(S) \leq c l V$,

(iii) $x \in \operatorname{\alpha int}\left(F^{+}(c l V)\right)$ for every fuzzy open set $V$ with $F(x) \leq V$,

(iv) $x \in \operatorname{int}\left(\operatorname{cl}\left(\operatorname{int}\left(F^{+}(\operatorname{cl} V)\right)\right)\right)$ for every fuzzy open set $V$ with $F(x) \leq V$.

Proof. (i) $\Rightarrow$ (ii) Let $x \in X$ and $V$ be any fuzzy open set in $Y$ with $F(x) \leq V$. By (i), for each $U \in S O(X, x)$, there exists a non empty open set $G_{U} \subset U$ such that $F\left(G_{U}\right) \leq c l V$. Let $W=\bigcup\left\{G_{U}: U \in S O(X, x)\right\}$. Then $W$ is open in $X$. Put $S=W \bigcup\{x\}$. Then $x \in \operatorname{scl} W$ and $F(W) \leq c l V$. Since $W \subseteq S \subseteq$ scl $W$ where $W$ is open in $X$, by Lemma 3.7(2), $S \in \alpha O(X, x)$. Also $F(S) \leq c l V$.

(ii) $\Rightarrow$ (iii) Let $V$ be any fuzzy open set in $Y$ with $F(x) \leq V$. By (ii), there exists $S \in \alpha O(X, x)$ such that $F(S) \leq c l V$. Then $x \in S \subseteq F^{+}(c l V) \Rightarrow x \in$ $S=\operatorname{\alpha int} S \subseteq \operatorname{\alpha int}\left(F^{+}(\operatorname{cl} V)\right)$.

(iii) $\Rightarrow$ (iv) Let $V$ be any fuzzy open set in $Y$ with $F(x) \leq V$. Then by (iii), $x \in \operatorname{\alpha int}\left(F^{+}(c l V)\right)$. Put $U=\operatorname{\alpha int}\left(F^{+}(c l V)\right)$. Then $U \in \alpha O(X, x)$ and so $x \in U \subseteq F^{+}(\operatorname{cl} V)$. Therefore, $x \in U \subseteq \operatorname{int}(\operatorname{cl}(\operatorname{int} U)) \subseteq \operatorname{int}\left(\operatorname{cl}\left(\operatorname{int}\left(F^{+}(\operatorname{cl} V)\right)\right)\right)$.

(iv) $\Rightarrow$ (i) Let $U \in S O(X, x)$ and $V$ be any fuzzy open set in $Y$ such that $F(x) \leq V$. By (iv), $x \in \operatorname{int}\left(\operatorname{cl}\left(\operatorname{int}\left(F^{+}(\operatorname{cl} V)\right)\right)\right)=\operatorname{scl}\left(\operatorname{int}\left(F^{+}(\operatorname{cl} V)\right)\right)$ (by Lemma $3.7(1))$. By definition, $U \bigcap \operatorname{int}\left(F^{+}(c l V)\right) \neq \phi$ and $U \bigcap \operatorname{int}\left(F^{+}(\operatorname{cl} V)\right) \in S O(X)$ (by Lemma 3 [18] and Lemma 1 [17]). Put $G=\operatorname{int}\left(U \bigcap \operatorname{int}\left(F^{+}(c l V)\right)\right)$. Then $G \neq \phi($ by Lemma $4[17]), G \subset U$ and $F(G) \leq c l V$.

Theorem 4.5. For a fuzzy multifunction $F: X \rightarrow$, the following statements are equivalent:

(i) $F$ is f.l.w. $\alpha$.c. at a point $x \in X$,

(ii) for any fuzzy open set $V$ with $F(x) q V$, there exists $U \in \alpha O(X, x)$ such that $F(u) q c l V$, for every $u \in U$,

(iii) $x \in \operatorname{\alpha int}\left(F^{-}(c l V)\right)$ for every fuzzy open set $V$ with $F(x) q V$,

(iv) $x \in \operatorname{int}\left(\operatorname{cl}\left(\operatorname{int}\left(F^{-}(c l V)\right)\right)\right)$ for every fuzzy open set $V$ with $F(x) q V$.

Proof. (i) $\Rightarrow$ (ii) Let $x \in X$ and $V$ be any fuzzy open set in $Y$ with $F(x) q V$. By (i), for each $U \in S O(X, x)$, there exists a non empty open set $G_{U} \subset U$ such that $F(g) q c l V$, for all $g \in G_{U}$. Put $W=\bigcup\left\{G_{U}: U \in S O(X, x)\right\}$. Then $W$ is open in $X$. Let $S=W \bigcup\{x\}$. Then $x \in \operatorname{scl} W$ and $F(w) q c l V$, for all $w \in W$. By Lemma 3.7(2), $S \in \alpha O(X, x)$ (as $W \subseteq S \subseteq s c l W$ ) and $F(s) q c l V$, for all 
$s \in S$.

(ii) $\Rightarrow$ (iii) Let $V$ be any fuzzy open set in $Y$ with $F(x) q V$. By (ii), there exists $U \in \alpha O(X, x)$ such that $F(u) q c l V$, for all $u \in U$. Then $x \in U \subseteq F^{-}(c l V)$ and hence $x \in U=\operatorname{\alpha int} U \subseteq \operatorname{\alpha int}\left(F^{-}(c l V)\right)$.

(iii) $\Rightarrow$ (iv) Let $V$ be any fuzzy open set in $Y$ with $F(x) q V$. Then by (iii), $x \in \operatorname{\alpha int}\left(F^{-}(\operatorname{cl} V)\right)$. Put $U=\operatorname{\alpha int}\left(F^{-}(c l V)\right)$. Then $U \in \alpha O(X, x)$ and so $x \in U \subseteq F^{-}(c l V)$. Thus $x \in U \subseteq \operatorname{int}(\operatorname{cl}(\operatorname{int} U)) \subseteq \operatorname{int}\left(\operatorname{cl}\left(\operatorname{int}\left(F^{-}(\operatorname{cl} V)\right)\right)\right)$.

(iv) $\Rightarrow$ (i) Let $U \in S O(X, x)$ and $V$ be any fuzzy open set in $Y$ such that $F(x) q V$. By (iv), $x \in \operatorname{int}\left(\operatorname{cl}\left(\operatorname{int}\left(F^{-}(\operatorname{cl} V)\right)\right)\right)=\operatorname{scl}\left(\operatorname{int}\left(F^{-}(\operatorname{cl} V)\right)\right)$ (by Lemma 3.7 (1)) and so $U \bigcap \operatorname{int}\left(F^{-}(c l V)\right) \neq \phi$ and $U \bigcap \operatorname{int}\left(F^{-}(c l V)\right) \in S O(X)$. Put $G=\operatorname{int}\left(U \bigcap \operatorname{int}\left(F^{-}(c l V)\right)\right)$. Then $G(\neq \phi)$ is open in $X$ with $G \subset U$ and $F(g) q c l V$, for all $g \in G$.

Theorem 4.6. For a fuzzy multifunction $F: X \rightarrow Y$, the following statements are equivalent:

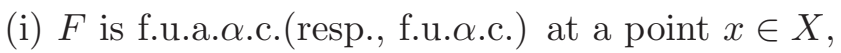

(ii) for any fuzzy open set $V$ of $Y$ with $F(x) \leq V$, there exists $S \in \alpha O(X, x)$ such that $F(S) \leq \operatorname{scl} V$ (resp., $F(S) \leq V$ ),

(iii) $x \in \operatorname{\alpha int}\left(F^{+}(\operatorname{scl} V)\right)$ (resp., $\left.x \in \operatorname{\alpha int}\left(F^{+}(V)\right)\right)$ for every fuzzy open set $V$ with $F(x) \leq V$,

(iv) $x \in \operatorname{int}\left(\operatorname{cl}\left(\operatorname{int}\left(F^{+}(\operatorname{scl} V)\right)\right)\right)$ (resp., $\left.x \in \operatorname{int}\left(\operatorname{cl}\left(\operatorname{int}\left(F^{+}(V)\right)\right)\right)\right)$ for every fuzzy open set $V$ with $F(x) \leq V$.

Proof. The proof is same as that of Theorem 4.4 .

Theorem 4.7. For a fuzzy multifunction $F: X \rightarrow Y$, the following statements are equivalent:

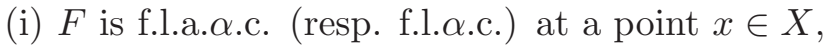

(ii) for any fuzzy open set $V$ with $F(x) q V$, there exists $U \in \alpha O(X, x)$ such that $F(u) q s c l V$ (resp., $F(u) q V$ ), for every $u \in U$,

(iii) $x \in \operatorname{\alpha int}\left(F^{-}(\operatorname{scl} V)\right)$ (resp., $\left.x \in \operatorname{\alpha int}\left(F^{-}(V)\right)\right)$ for every fuzzy open set $V$ with $F(x) q V$,

(iv) $x \in \operatorname{int}\left(\operatorname{cl}\left(\operatorname{int}\left(F^{-}(\operatorname{cl} V)\right)\right)\right)$ (resp., $\left.x \in \operatorname{int}\left(\operatorname{cl}\left(\operatorname{int}\left(F^{-}(V)\right)\right)\right)\right)$ for every fuzzy open set $V$ with $F(x) q V$.

Proof. The proof is same as that of Theorem 4.5.

Theorem 4.8. For a fuzzy multifunction $F: X \rightarrow Y$, the following statements are equivalent:

(i) $F$ is f.u.w.o.c.,

(ii) for each $x \in X$ and each fuzzy open set $V$ of $Y$ with $F(x) \leq V$, there exists $U \in \alpha O(X, x)$ such that $F(U) \leq c l V$,

(iii) $F^{+}(V) \subseteq \operatorname{int}\left(\operatorname{cl}\left(\operatorname{int}\left(F^{+}(\operatorname{cl} V)\right)\right)\right)$ for every fuzzy open set $V$ of $Y$, 
(iv) $\operatorname{cl}\left(\operatorname{int}\left(\operatorname{cl}\left(F^{-}(\operatorname{int} K)\right)\right)\right) \subseteq F^{-}(K)$ for every fuzzy closed set $V$ of $Y$,

(v) $\alpha \operatorname{cl}\left(F^{-}(\right.$int $\left.K)\right) \subseteq F^{-}(K)$ for every fuzzy closed set $K$ of $Y$,

(vi) $\alpha c l\left(F^{-}(\operatorname{int}(c l B))\right) \subseteq F^{-}(\operatorname{cl} B)$ for every fuzzy set $B$ of $Y$,

(vii) $F^{+}($int $B) \subseteq \operatorname{\alpha int}\left(F^{+}(\operatorname{cl}(\right.$ int $\left.B))\right)$ for every fuzzy set $B$ of $Y$,

(viii) $F^{+}(V) \subseteq \operatorname{\alpha int}\left(F^{+}(c l V)\right)$ for every fuzzy open set $V$ of $Y$,

(ix) $\alpha c l\left(F^{-}(\right.$int $\left.K)\right) \subseteq F^{-}(K)$ for every fuzzy regular closed set $K$ of $Y$,

(x) $\alpha c l\left(F^{-}(V)\right) \subseteq F^{-}(c l V)$ for every fuzzy open set $V$ of $Y$,

(xi) $\alpha c l\left(F^{-}(\operatorname{int}(\theta c l B))\right) \subseteq F^{-}(\theta c l B)$ for every fuzzy set $B$ of $Y$.

Proof. (i) $\Rightarrow$ (ii) Follows from Theorem 4.4.

(ii) $\Rightarrow$ (iii) Let $x \in X$ and $V$ be any fuzzy open set in $Y$ with $x \in F^{+}(V)$. Then $F(x) \leq V$. By (ii), there exists $U \in \alpha O(X, x)$ such that $F(U) \leq c l V$. Therefore, $x \in U \subseteq F^{+}(c l V)$. Since $\left.U \in \alpha O(X, x), x \in U \subseteq \operatorname{int}(\operatorname{cl}(\operatorname{int} U))\right) \subseteq$ $\operatorname{int}\left(\operatorname{cl}\left(\operatorname{int}\left(F^{+}(\operatorname{cl} V)\right)\right)\right) \Rightarrow F^{+}(V) \subseteq \operatorname{int}\left(\operatorname{cl}\left(\left(\operatorname{int}\left(F^{+}(\operatorname{cl} V)\right)\right)\right)\right.$.

(iii) $\Rightarrow$ (iv) Let $K$ be a fuzzy closed set of $Y$. Then $1_{Y} \backslash K$ is fuzzy open in $Y$. By (iii),

$$
\begin{aligned}
& F^{+}\left(1_{Y} \backslash K\right) \subseteq \operatorname{int}\left(\operatorname{cl}\left(\operatorname{int}\left(F^{+}\left(\operatorname{cl}\left(1_{Y} \backslash K\right)\right)\right)\right)\right) \\
& \Rightarrow X \backslash F^{-}(K) \subseteq \operatorname{int}\left(\operatorname{cl}\left(\operatorname{int}\left(X \backslash F^{-}(\operatorname{int} K)\right)\right)\right)=X \backslash \operatorname{cl}\left(\operatorname{int}\left(\operatorname{cl}\left(F^{-}(\operatorname{int} K)\right)\right)\right) \\
& \Rightarrow \operatorname{cl}\left(\operatorname{int}\left(\operatorname{cl}\left(F^{-}(\operatorname{int} K)\right)\right)\right) \subseteq F^{-}(K) .
\end{aligned}
$$

(iv) $\Rightarrow(\mathrm{v})$ Let $K$ be a fuzzy closed set of $Y$. By (iv), $\operatorname{cl}\left(\operatorname{int}\left(\operatorname{cl}\left(F^{-}(\right.\right.\right.$int $\left.\left.\left.K)\right)\right)\right) \subseteq$ $F^{-}(K)$ and hence by Lemma $3.7(3), \alpha c l\left(F^{-}(\right.$int $\left.K)\right) \subseteq F^{-}(K)$.

$(\mathrm{v}) \Rightarrow(\mathrm{vi})$ Let $B$ be a fuzzy set of $Y$. Then $\mathrm{cl} B$ is fuzzy closed in $Y$ and so by $(\mathrm{v}), \alpha c l\left(F^{-}(\operatorname{int}(\operatorname{cl} B))\right) \subseteq F^{-}(\operatorname{cl} B)$.

(vi) $\Rightarrow$ (vii) Let $B$ be a fuzzy set of $Y$. Then $X \backslash F^{+}($int $B)=F^{-}\left(1_{Y} \backslash\right.$ $\operatorname{int} B)=F^{-}\left(\operatorname{cl}\left(1_{Y} \backslash B\right)\right) \supseteq \alpha \operatorname{cl}\left(F^{-}\left(\operatorname{int}\left(\operatorname{cl}\left(1_{Y} \backslash B\right)\right)\right)=\alpha \operatorname{cl}\left(F^{-}\left(1_{Y} \backslash \operatorname{cl}(\operatorname{int} B)\right)\right)=\right.$ $\alpha c l\left(X \backslash F^{+}(\operatorname{cl}(\operatorname{int} B))\right)=X \backslash \operatorname{\alpha int}^{+}(\operatorname{cl}(\operatorname{int} B)) \Rightarrow \operatorname{\alpha int}\left(F^{+}(\operatorname{cl}(\operatorname{int} B))\right) \supseteq$ $F^{+}($int $B)$.

(vii) $\Rightarrow$ (viii) Obvious.

(viii) $\Rightarrow$ (i) Let $x \in X$ and $V$ be any fuzzy open set in $Y$ such that $F(x) \leq V$. Then $x \in F^{+}(V) \subseteq \operatorname{\alpha int}\left(F^{+}(\operatorname{cl} V)\right)$ (by (viii) $) \subseteq \operatorname{int}\left(\operatorname{cl}\left(\operatorname{int}\left(F^{+}(\operatorname{cl} V)\right)\right)\right)$ (by

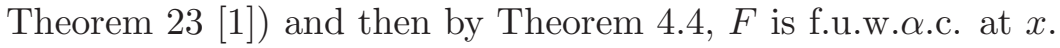

$(\mathrm{v}) \Rightarrow(\mathrm{ix})$ Obvious.

$(\mathrm{ix}) \Rightarrow(\mathrm{x})$ Let $V$ be any fuzzy open set of $Y$. Then $c l V$ is fuzzy regular closed in $Y$. Indeed, $\operatorname{cl}(\operatorname{int}(\operatorname{cl} V)) \leq \operatorname{cl} V$ and $c l V=\operatorname{cl}(\operatorname{int} V) \leq \operatorname{cl}(\operatorname{int}(\operatorname{cl} V))$. By (ix), $\alpha c l\left(F^{-}(\operatorname{int}(c l V))\right) \subseteq F^{-}(\operatorname{cl} V) \Rightarrow \alpha c l\left(F^{-}(V)\right) \subseteq \alpha c l\left(F^{-}(\operatorname{int}(c l V)) \subseteq F^{-}(c l V)\right.$.

$(\mathrm{x}) \Rightarrow$ (viii) Let $V$ be any fuzzy open set of $Y$. By $(\mathrm{x}), \alpha c l\left(F^{-}(V)\right) \subseteq$ $F^{-}(c l V)$. Therefore, $X \backslash \alpha \operatorname{\alpha int}\left(F^{+}(c l V)\right)=\alpha c l\left(X \backslash F^{+}(c l V)\right)=\alpha c l\left(F^{-}\left(1_{Y} \backslash\right.\right.$ 
$c l V)) \subseteq F^{-}\left(c l\left(1_{Y} \backslash c l V\right)\right)=F^{-}\left(1_{Y} \backslash \operatorname{int}(c l V)\right)=X \backslash F^{+}(\operatorname{int}(c l V))$ and hence $F^{+}(V) \subseteq F^{+}(\operatorname{int}(c l V)) \subseteq \operatorname{\alpha int}\left(F^{+}(c l V)\right)$.

$(\mathrm{x}) \Rightarrow$ (xi) Let $B$ be any fuzzy set of $Y$. Put $V=\operatorname{int}(\theta c l B)$. Then $V$ is fuzzy open in $Y$. By $(\mathrm{x}), \alpha c l\left(F^{-}(\operatorname{int}(\theta \operatorname{cl} B))\right) \subseteq F^{-}(\operatorname{cl}(\operatorname{int}(\theta \operatorname{cl} B))) \subseteq F^{-}(\operatorname{cl}(\theta \operatorname{cl} B))=$ $F^{-}(\theta c l B)$ (as $\theta c l B$ is fuzzy closed in $\left.Y[16]\right)$.

$(\mathrm{xi}) \Rightarrow$ (ix) Let $K$ be any fuzzy regular closed set of $Y$. Then by (xi),

$$
\begin{array}{r}
\alpha c l\left(F^{-}(\operatorname{int} K)\right)=\alpha c l\left(F^{-}(\operatorname{int}(\operatorname{cl} K))\right)=\alpha c l\left(F^{-}(\operatorname{int}(\operatorname{cl}(\operatorname{cl}(\operatorname{int} K))))\right) \\
=\alpha c l\left(F^{-}(\operatorname{int}(\operatorname{cl}(\operatorname{int} K)))\right)=\alpha c l\left(F^{-}(\operatorname{int}(\theta \operatorname{cl}(\operatorname{int} K)))\right)
\end{array}
$$

(since int $K$ is fuzzy open in $Y) \subseteq \alpha \operatorname{cl}\left(F^{-}(\theta \operatorname{cl}(\right.$ int $\left.K))\right) \subseteq F^{-}(\theta \operatorname{cl}($ int $K))$ (by $(\mathrm{xi}))=F^{-}(c l($ int $K))($ as int $K$ is fuzzy open in $Y)=F^{-}(K)$.

Theorem 4.9. For a fuzzy multifunction $F: X \rightarrow Y$, the following statements are equivalent:

(i) $F$ is f.l.w.Q.c.,

(ii) for each $x \in X$ and each fuzzy open set $V$ of $Y$ with $F(x) q V$, there exists $U \in \alpha O(X, x)$ such that $U \subseteq F^{-}(c l V)$, i.e., $F(u) q c l V$, for all $u \in U$,

(iii) $F^{-}(V) \subseteq \operatorname{int}\left(\operatorname{cl}\left(\operatorname{int}\left(F^{-}(V)\right)\right)\right)$ for every fuzzy open set $V$ of $Y$,

(iv) $\operatorname{cl}\left(\operatorname{int}\left(\operatorname{cl}\left(F^{+}(\operatorname{int} K)\right)\right)\right) \subseteq F^{+}(K)$ for every fuzzy closed set $V$ of $Y$,

(v) $\alpha c l\left(F^{+}(\right.$int $\left.K)\right) \subseteq F^{+}(K)$ for every fuzzy closed set $K$ of $Y$,

(vi) $\alpha c l\left(F^{+}(\operatorname{int}(\operatorname{cl} B))\right) \subseteq F^{+}(\operatorname{cl} B)$ for every fuzzy set $B$ of $Y$,

(vii) $F^{-}($int $B) \subseteq \operatorname{\alpha int}\left(F^{-}(\operatorname{cl}(\right.$ int $\left.B))\right)$ for every fuzzy set $B$ of $Y$,

(viii) $F^{-}(V) \subseteq \operatorname{\alpha int}\left(F^{-}(c l V)\right)$ for every fuzzy open set $V$ of $Y$,

(ix) $\alpha c l\left(F^{+}(\right.$int $\left.K)\right) \subseteq F^{+}(K)$ for every fuzzy regular closed set $K$ of $Y$,

(x) $\alpha c l\left(F^{+}(V)\right) \subseteq F^{+}(c l V)$ for every fuzzy open set $V$ of $Y$,

(xi) $\alpha c l\left(F^{+}(\operatorname{int}(\theta c l B))\right) \subseteq F^{+}(\theta c l B)$ for every fuzzy set $B$ of $Y$.

PROOF. (i) $\Rightarrow$ (ii) Follows from Theorem 4.5.

(ii) $\Rightarrow$ (iii) Let $x \in X$ and $V$ be any fuzzy open set in $Y$ with $x \in F^{-}(V)$. Then $F(x) \leq V$. By (ii), there exists $U \in \alpha O(X, x)$ such that $U \subseteq F^{-}(c l V)$. Therefore, $x \in U \subseteq \operatorname{int}(\operatorname{cl}(\operatorname{int} U))) \subseteq \operatorname{int}\left(\operatorname{cl}\left(\operatorname{int}\left(F^{-}(\operatorname{cl} V)\right)\right)\right) \Rightarrow F^{-}(V) \subseteq$ $\operatorname{int}\left(\operatorname{cl}\left(\left(\operatorname{int}\left(F^{-}(c l V)\right)\right)\right)\right.$.

(iii) $\Rightarrow$ (iv) Let $K$ be a fuzzy closed set of $Y$. Then $1_{Y} \backslash K$ is fuzzy open in $Y$. By (iii),

$$
\begin{aligned}
& F^{-}\left(1_{Y} \backslash K\right) \subseteq \operatorname{int}\left(\operatorname{cl}\left(\operatorname{int}\left(F^{-}\left(\operatorname{cl}\left(1_{Y} \backslash K\right)\right)\right)\right)\right) \\
& \Rightarrow X \backslash F^{+}(K) \subseteq \operatorname{int}\left(\operatorname{cl}\left(\operatorname{int}\left(X \backslash F^{+}(\operatorname{int} K)\right)\right)\right)=X \backslash \operatorname{cl}\left(\operatorname{int}\left(\operatorname{cl}\left(F^{+}(\operatorname{int} K)\right)\right)\right) \\
& \Rightarrow \operatorname{cl}\left(\operatorname{int}\left(\operatorname{cl}\left(F^{+}(\operatorname{int} K)\right)\right)\right) \subseteq F^{+}(K) .
\end{aligned}
$$


(iv) $\Rightarrow$ (v) Let $K$ be a fuzzy closed set of $Y$. By (iv), $\operatorname{cl}\left(\operatorname{int}\left(\operatorname{cl}\left(F^{+}(\right.\right.\right.$int $\left.\left.\left.K)\right)\right)\right) \subseteq$ $F^{+}(K)$ and hence by Lemma $3.7(3)$,

$$
\begin{aligned}
\alpha \operatorname{cl}\left(F^{+}(\operatorname{int} K)\right)=F^{+}((\operatorname{int} K) \bigcup \operatorname{cl}(\operatorname{int}( & \left.\operatorname{cl}\left(F^{+}(\operatorname{int} K)\right)\right) \\
& \subseteq F^{+}(\operatorname{intK}) \bigcup F^{+}(K) \subseteq F^{+}(K)
\end{aligned}
$$

(as $F^{+}($int $\left.K) \subseteq F^{+}(K)\right)$.

(v) $\Rightarrow$ (vi) Let $B$ be a fuzzy set of $Y$. Then $c l B$ is fuzzy closed in $Y$ and so by $(\mathrm{v}), \alpha c l\left(F^{+}(\operatorname{int}(c l B))\right) \subseteq F^{+}(c l B)$.

(vi) $\Rightarrow$ (vii) Let $B$ be any fuzzy set of $Y$. Then $X \backslash F^{-}($int $B)=F^{+}\left(1_{Y} \backslash\right.$ int $B)=F^{+}\left(\operatorname{cl}\left(1_{Y} \backslash B\right)\right) \supseteq \alpha \operatorname{cl}\left(F^{+}\left(\operatorname{int}\left(\operatorname{cl}\left(1_{Y} \backslash B\right)\right)\right)=\alpha c l\left(F^{+}\left(1_{Y} \backslash \operatorname{cl}(\operatorname{int} B)\right)\right)=\right.$ $\alpha c l\left(X \backslash F^{-}(\operatorname{cl}(\operatorname{int} B))\right)=X \backslash \operatorname{\alpha int}^{-}(\operatorname{cl}(\operatorname{int} B)) \Rightarrow \operatorname{\alpha int}\left(F^{-}(\operatorname{cl}(\operatorname{int} B))\right) \supseteq$ $F^{-}($int $B)$.

(vii) $\Rightarrow$ (viii) Obvious.

(viii) $\Rightarrow$ (i) Let $x \in X$ and $V$ be any fuzzy open set in $Y$ such that $F(x) q V$. Then $x \in F^{-}(V) \subseteq \operatorname{\alpha int}\left(F^{-}(c l V)\right)$ (by (viii) $\subseteq \operatorname{int}\left(\operatorname{cl}\left(\operatorname{int}\left(F^{-}(c l V)\right)\right)\right.$ ) (by

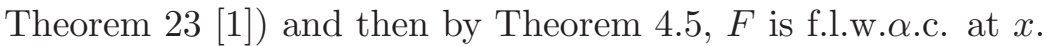

(v) $\Rightarrow$ (ix) The proof follows from the fact that every fuzzy regular closed set is fuzzy closed.

(ix) $\Rightarrow(\mathrm{x})$ Let $V$ be any fuzzy open set of $Y$. Then $c l V$ is fuzzy regular closed in $Y$. By (ix), $\alpha c l\left(F^{+}(\operatorname{int}(\operatorname{cl} V))\right) \subseteq F^{+}(\operatorname{clV}) \Rightarrow \alpha c l\left(F^{+}(V)\right) \subseteq$ $\alpha c l\left(F^{+}(\operatorname{int}(c l V)) \subseteq F^{+}(c l V)\right.$.

$(\mathrm{x}) \Rightarrow$ (viii) Let $V$ be any fuzzy open set of $Y$. By (x), $\alpha c l\left(F^{+}(V)\right) \subseteq$ $F^{+}(c l V)$. Therefore, $X \backslash \alpha \operatorname{\alpha int}\left(F^{-}(c l V)\right)=\alpha c l\left(X \backslash F^{-}(c l V)\right)=\alpha c l\left(F^{+}\left(1_{Y} \backslash\right.\right.$ $c l V)) \subseteq F^{+}\left(c l\left(1_{Y} \backslash c l V\right)\right)=F^{+}\left(1_{Y} \backslash \operatorname{int}(c l V)\right)=X \backslash F^{-}(\operatorname{int}(c l V))$ and hence $F^{-}(V) \subseteq F^{-}(\operatorname{int}(c l V)) \subseteq \operatorname{\alpha int}\left(F^{-}(c l V)\right)$.

$(\mathrm{x}) \Rightarrow(\mathrm{xi})$ Let $B$ be any fuzzy set of $Y$. Put $V=\operatorname{int}(\theta \operatorname{cl} B)$. Then $V$ is fuzzy open in $Y$. By $(\mathrm{x}), \alpha c l\left(F^{+}(V)\right) \subseteq F^{+}(\operatorname{clV}) \Rightarrow \alpha \operatorname{\alpha cl}\left(F^{+}(\operatorname{int}(\theta \operatorname{cl} B))\right) \subseteq$ $F^{+}(\operatorname{cl}(\operatorname{int}(\theta \operatorname{cl} B))) \subseteq F^{+}(\operatorname{cl}(\theta \operatorname{cl} B))=F^{+}(\theta \operatorname{cl} B)$ (as $\theta \operatorname{cl} B$ is fuzzy closed in $Y$ [16]).

$(\mathrm{xi}) \Rightarrow(\mathrm{ix})$ Let $K$ be any fuzzy regular closed set of $Y$. Then, $\alpha c l\left(F^{+}(\right.$int $\left.K)\right)=$ $\alpha c l\left(F^{+}(\operatorname{int}(\operatorname{cl} K))\right)=\alpha c l\left(F^{+}(\operatorname{int}(\operatorname{cl}(\operatorname{cl}(\operatorname{int} K))))\right)=\alpha c l\left(F^{+}(\operatorname{int}(\operatorname{cl}(\operatorname{int} K)))\right)=$ $\alpha c l\left(F^{+}(\operatorname{int}(\theta \operatorname{cl}(\right.$ int $\left.K)))\right)($ since $\operatorname{int} K$ is fuzzy open in $Y) \subseteq \alpha c l\left(F^{+}(\theta \operatorname{cl}(\right.$ int $\left.K))\right) \subseteq$ $F^{+}(\theta \operatorname{cl}($ int $K))($ by $(\mathrm{xi}))=F^{+}(\operatorname{cl}(\operatorname{int} K))($ as int $K$ is fuzzy open in $Y)=F^{+}(K)$.

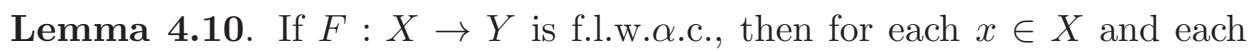
fuzzy set $B$ of $Y$ with $F(x) q(\theta i n t B)$, there exists $U \in \alpha O(X, x)$ such that $U \subseteq F^{-}(B)$.

Proof. Let $F$ be f.l.w. $\alpha . c$. and $x \in X$ and $B$ be fuzzy open in $Y$ with $F(x) q(\theta$ int $B)$. Then there exists $y \in Y$ such that $[F(x)](y)+(\theta$ int $B)(y)>1$. 
Let $[F(x)](y)=t$. Then $(\theta$ int $B)(y)+t>1 \Rightarrow y_{t} q(\theta$ int $B)$ and $t>1-$ $(\theta \operatorname{int} B)(y) \Rightarrow y_{t} \notin 1_{Y} \backslash \theta \operatorname{int} B=\theta \operatorname{cl}\left(1_{Y} \backslash B\right)$. Then there exists a fuzzy open set $V$ in $Y$ with $y_{t} q V, c l V / q\left(1_{Y} \backslash B\right) \Rightarrow c l V \leq B$. Therefore, $F(x) q V$ (as

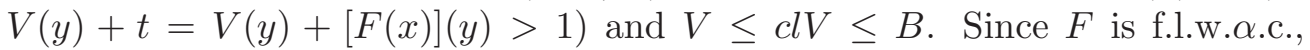
there exists $U \in \alpha O(X, x)$ such that $F(u) q c l V$, for all $u \in U \Rightarrow F(u) q B$, for all $u \in U \Rightarrow U \subseteq F^{-}(B)$.

Theorem 4.11. For a fuzzy multifunction $F: X \rightarrow Y$, the following statements are equivalent:

(i) $F$ is f.l.w.Q.c.,

(ii) $\alpha c l\left(F^{+}(B)\right) \subseteq F^{+}(\theta c l B)$, for every fuzzy set $B$ of $Y$,

(iii) $F(\alpha c l A) \leq \theta \operatorname{cl}(F(A))$, for every subset $A$ of $X$.

Proof. (i) $\Rightarrow$ (ii) Let $B$ be a fuzzy set of $Y$ and $x \notin F^{+}(\theta \operatorname{cl} B)$. Then $x \in X \backslash F^{+}(\theta c l B)=F^{-}\left(1_{Y} \backslash \theta c l B\right)=F^{-}\left(\theta i n t\left(1_{Y} \backslash B\right)\right)$. By Lemma 4.10, there exists $U \in \alpha O(X, x)$ such that $U \subseteq F^{-}\left(1_{Y} \backslash B\right)=X \backslash F^{+}(B)$ and so $U \cap F^{+}(B)=\phi$. Again, $F^{+}(B) \subseteq \alpha c l\left(F^{+}(B)\right) \Rightarrow X \backslash F^{+}(B) \supseteq X \backslash$ $\alpha c l\left(F^{+}(B)\right)$. Now $F^{+}(B) \subseteq X \backslash U \Rightarrow \alpha c l\left(F^{+}(B)\right) \subseteq \alpha c l(X \backslash U)=X \backslash U \Rightarrow$ $U \subseteq X \backslash \alpha \operatorname{cl}\left(F^{+}(B)\right) \Rightarrow x \in U \subseteq X \backslash \alpha \operatorname{cl}\left(F^{+}(B)\right) \Rightarrow x \notin \alpha c l\left(F^{+}(B)\right)$.

(ii) $\Rightarrow$ (i) Let $V$ be a fuzzy open set of $Y$. Then $c l V=\theta c l V$. By (ii), $\alpha c l\left(F^{+}(V)\right) \subseteq F^{+}(\theta c l V)=F^{+}(c l V)$ and so by Theorem $4.9(\mathrm{x}) \Rightarrow(\mathrm{i}), F$ is f.l.w.Q.c.

(ii) $\Rightarrow$ (iii) Let $A \subseteq X$. By (ii), $\alpha c l A \subseteq \alpha c l\left(F^{+}(F(A))\right) \subseteq F^{+}(\theta \operatorname{cl}(F(A)))$ and so $F(\alpha c l A) \leq \theta \operatorname{cl}(F(A))$.

(iii) $\Rightarrow$ (ii) Let $B$ be a fuzzy set of $Y$. Then $F^{+}(B) \subseteq X$. By (iii), $F\left(\alpha c l\left(F^{+}(B)\right)\right) \leq \theta \operatorname{cl}\left(F\left(F^{+}(B)\right)\right) \leq \theta \operatorname{cl} B \Rightarrow \alpha c l\left(F^{+}(B)\right) \subseteq F^{+}(\theta \operatorname{cl} B)$.

Let us now recall the following definition and lemmas from [4] for ready references.

Definition 4.12. For a fuzzy multifunction $F: X \rightarrow Y, c l F: X \rightarrow Y$ and $\alpha c l F: X \rightarrow Y$ are defined by $(\operatorname{cl} F)(x)=\operatorname{cl} F(x)$, for all $x \in X$ [3] and $(\alpha c l F)(x)=\alpha c l F(x)$, for all $x \in X[4]$.

Lemma 4.13 [4]. For a fuzzy multifunction $F: X \rightarrow Y,(c l F)^{-}(V)=$ $F^{-}(V),(\alpha c l F)^{-}(V)=F^{-}(V)$, for all fuzzy open set $V$ in $Y$.

Lemma 4.14 [4]. For a fuzzy multifunction $F: X \rightarrow Y,(\alpha c l F)^{+}(V)=$ $F^{+}(V)$, for all fuzzy set $V$ in $Y$.

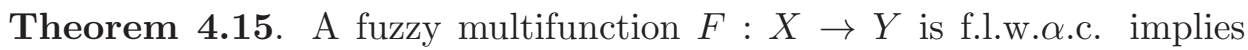

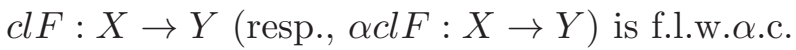

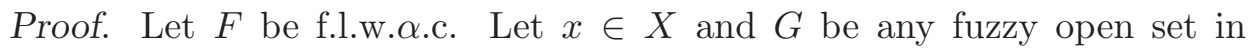
$Y$ such that $(c l F)(x) q G$ (resp., $(\alpha c l F)(x) q G)$. Then $x \in(c l F)^{-}(G)$ (resp., 
$\left.x \in(\alpha c l F)^{-}(G)\right)=F^{-}(G)$ (by Lemma 4.13). As $F$ is f.l.w. $\alpha . c$. , there exists $U \in \alpha O(X, x)$ such that $F(u) q c l G$, for all $u \in U \Rightarrow(c l F)(u) q c l G$ (resp., $(\alpha c l F)(u) q c l G)$, for all $u \in U \Rightarrow u \in(c l F)^{-}(c l G)$ (resp., $\left.u \in(\alpha c l F)^{-}(c l G)\right)$, for all $u \in U \Rightarrow U \subseteq(c l F)^{-}(\operatorname{cl} G)$ (resp., $\left.U \subseteq(\alpha c l F)^{-}(c l G)\right) \Rightarrow c l F$ (resp., $\alpha c l F)$ is f.l.w. $\alpha . c$.

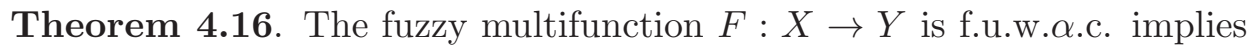
$\alpha c l F: X \rightarrow Y$ is f.u.w. $\alpha . c$.

Proof. Let $x \in X$ and $G$ be any fuzzy open set in $Y$ such that $(\alpha c l F)(x) \leq$

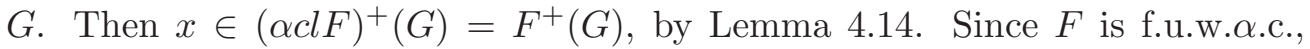
there exists $U \in \alpha O(X, x)$ such that $F(U) \leq \operatorname{cl} G \Rightarrow(\alpha c l F)(U) \leq \alpha c l(c l G) \leq$ $c l(c l G)=c l G \Rightarrow \alpha c l F$ is f.u.w. $\alpha$.c.

Definition 4.17 [14]. For a fuzzy multifunction $F: X \rightarrow Y$, the fuzzy graph multifunction $G_{F}: X \rightarrow X \times Y$ of $F$ is defined as $G_{F}(x)=$ the fuzzy set $x_{1} \times F(x)$ of $X \times Y$, where $x_{1}$ is the fuzzy set in $X$, whose value is 1 at $x \in X$ and 0 at other points of $X$. We shall write $\{x\} \times F(x)$ for $x_{1} \times F(x)$.

Lemma 4.18 [3]. The following hold for a fuzzy multifunction $F: X \rightarrow Y$ :

(a) $\left(G_{F}\right)^{+}(A \times B)=A \bigcap F^{+}(B)$ and

(b) $\left(G_{F}\right)^{-}(A \times B)=A \bigcap F^{-}(B)$ for every subset $A$ of $X$ and every fuzzy set $B$ of $Y$.

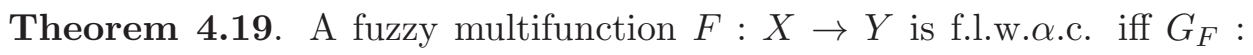
$X \rightarrow X \times Y$ is f.l.w. $\alpha . c$.

Proof. Let $G_{F}$ be f.l.w. $\alpha . c$. on $X$. Let $x \in X$ and $V$ be any fuzzy open set in $Y$ with $F(x) q V$. Then there exists $y \in Y$ such that $[F(x)](y)+V(y)>1$. Now $1_{X} \times V$ is fuzzy open in $X \times Y$ such that $[\{x\} \times F(x)](x, y)+\left(1_{X} \times V\right)(x, y)>1$ and so $G_{F}(x) q\left(1_{X} \times V\right)$. Since $G_{F}$ is f.l.w. $\alpha . c$., there exists $U \in \alpha O(X, x)$ such that $\left(G_{F}\right)(u) q c l\left(1_{X} \times V\right)$, for all $u \in U \Rightarrow U \subseteq\left(G_{F}\right)^{-}\left(1_{X} \times c l V\right)=$ $1_{X} \cap F^{-}(c l V)=F^{-}(c l V) \Rightarrow F$ is f.l.w. $\alpha . c$. on $X$.

Conversely, let $F$ be f.l.w. $\alpha . c$. Let $x \in X$ and $W$ be any fuzzy open set of $X \times Y$ such that $G_{F}(x) q W$. Then there exists $(x, y) \in X \times Y$ such that $[\{x\} \times F(x)](y)+W(x, y)>1$. Then $[F(x)](y)+W(x, y)>1$. Let $[F(x)](y)=$ $\alpha$. Then $W(x, y)+\alpha>1 \Rightarrow(x, y)_{\alpha} q W$ and hence $(x, y)_{\alpha} q(U \times V) \leq W$ for some open set $U$ of $X$ and a fuzzy open set $V$ of $Y$ with $y_{\alpha} q V$. Then

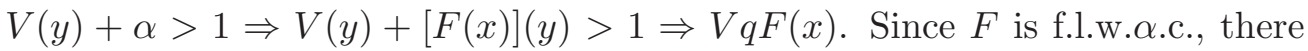
exists $G \in \alpha O(X, x)$ such that $F(g) q c l V$, for all $g \in G \Rightarrow G \subseteq F^{-}(c l V)$. Then by Lemma 2.13(b), $U \cap G \subseteq U \cap F^{-}(c l V)=\left(G_{F}\right)^{-}(U \times c l V) \subseteq\left(G_{F}\right)^{-}(c l W)$. Also $x \in U \cap G \in \alpha O(X, x)$ which proves that $G_{F}$ is f.l.w. $\alpha . c$.

Theorem 4.20. When $X$ is product related to $Y$, a fuzzy multifunction $F: X \rightarrow Y$ is f.u.w. $\alpha . c$. if its fuzzy graph multifunction $G_{F}: X \rightarrow X \times Y$ is 


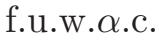

Proof. Let $G_{F}$ be f.u.w. $\alpha$.c. on $X$. Let $x \in X$ and $V$ be any fuzzy open set in $Y$ with $F(x) \leq V$. Then $G_{F}(x) \leq 1_{X} \times V$ and $1_{X} \times V$ is open in $X \times V$. Then there exists $U \in \alpha O(X, x)$ such that $G_{F}(U) \leq \operatorname{cl}\left(1_{X} \times V\right)=1_{X} \times c l V$. Now for any $z \in U$ and for any $y \in Y,[F(z)](y)=\left[G_{F}(z)\right](z, y) \leq\left(1_{X} \times c l V\right)(z, y)=$ $(c l V)(y)$, i.e., $[F(z)](y) \leq(c l V)(y)$, for all $y \in Y \Rightarrow F(z) \leq c l V$, for any

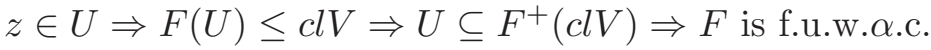

Lemma 4.21 [13]. Let $U, X_{0} \subseteq X$. Then the following properties hold:

(i) if $U \in \alpha O(X)$ and $X_{0} \in S O(X) \cup P O(X)$, then $U \cap X_{0} \in \alpha O\left(X_{0}\right)$,

(ii) if $U \subseteq X_{0} \subseteq X, U \in \alpha O\left(X_{0}\right)$ and $X_{0} \in \alpha O(X)$, then $U \in \alpha O(X)$.

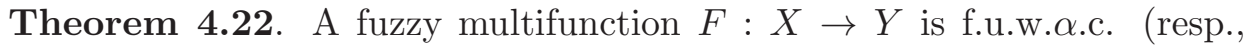

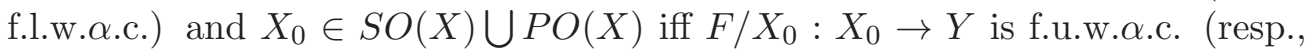
f.l.w. $\alpha . c$.$) .$

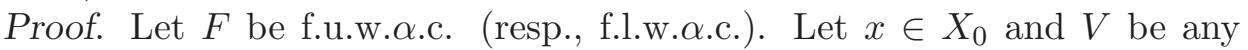
fuzzy open set in $Y$ such that $\left(F / X_{0}\right)(x) \leq V$ (resp., $\left.\left(F / X_{0}\right)(x) q V\right)$. Then $F(x) \leq V$ (resp., $F(x) q V)$. By hypothesis, there exists $U \in \alpha O(X, x)$ such that $F(U) \leq c l V$ (resp., $F(u) q c l V$, for all $u \in U$ ). Let $U_{0}=U \cap X_{0}$. Then by Lemma $4.21(\mathrm{i}), U_{0} \in \alpha O\left(X_{0}, x\right)$ and $\left(F / X_{0}\right)\left(U_{0}\right)=F\left(U_{0}\right) \leq c l V$ (resp.,

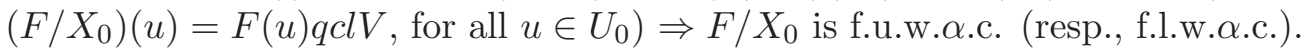

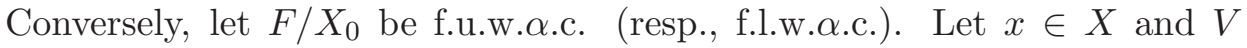
be any fuzzy open set in $Y$ such that $F(x) \leq V$ (resp., $F(x) q V$ ). Then

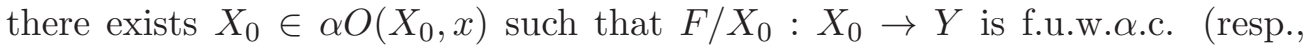

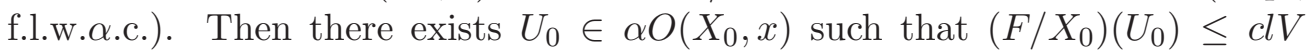
(resp., $\left(F / X_{0}\right)(u) q c l V$, for all $\left.u \in U_{0}\right)$. By Lemma 4.21(ii), $U_{0} \in \alpha O(X, x)$

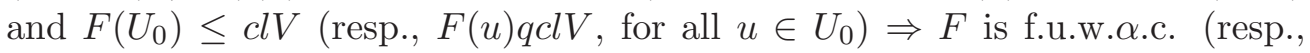
f.l.w.o.c.).

\section{Mutual Relationships}

It is clear from Definition 4.1, Definition 4.2 and Definition 4.3 that f.u. $\alpha . c . \Rightarrow$

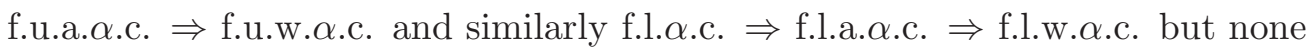
of the above implications are reversible as seen from the following examples.

Example 5.1. Let $X=\{a, b\}, \tau_{X}=\{\phi, X\}, Y=[0,1], \tau_{Y}=\left\{0_{Y}, 1_{Y}, A, B\right\}$ where $A(y)=0.35, B(y)=0.4$, for all $y \in Y$. Then $\left(X, \tau_{X}\right)$ and $\left(Y, \tau_{Y}\right)$ are a topological space and an fts respectively. Let $F:\left(X, \tau_{X}\right) \rightarrow\left(Y, \tau_{Y}\right)$ be a fuzzy multifunction defined by $F(a)=B, F(b)=C$ where $C(y)=0.6$, for all $y \in Y$. Now $F^{+}(A)=\phi, F^{+}(B)=\{a\}$ and $F^{+}(c l B)=F^{+}\left(1_{Y} \backslash B\right)=X$. 
Again $\operatorname{int}\left(\operatorname{cl}\left(\operatorname{int}\left(F^{+}(\operatorname{cl} B)\right)\right)\right)=X \Rightarrow F^{+}(B) \subseteq \operatorname{int}\left(\operatorname{cl}\left(\operatorname{int}\left(F^{+}(\operatorname{clB})\right)\right)\right) \Rightarrow F$ is

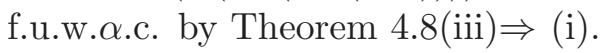

But $\operatorname{int}\left(\operatorname{cl}\left(\operatorname{int}\left(F^{+}(B)\right)\right)\right)=\operatorname{int}(\operatorname{cl}(\operatorname{int}(\{a\})))=\operatorname{int}(\operatorname{cl}(\operatorname{int}(\phi)))=\phi \Rightarrow$

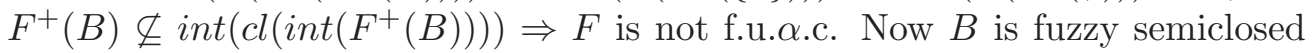
in $Y$ as $\operatorname{int}(\operatorname{cl} B)=\operatorname{int}\left(1_{Y} \backslash B\right)=B$ and so

$$
\begin{gathered}
\operatorname{int}\left(\operatorname{cl}\left(\operatorname{int}\left(F^{+}(\operatorname{scl} B)\right)\right)\right)=\operatorname{int}\left(\operatorname{cl}\left(\operatorname{int}\left(F^{+}(B)\right)\right)\right)=\operatorname{int}(\operatorname{cl}(\operatorname{int}(\{a\})))=\phi \\
\Rightarrow F^{+}(B) \nsubseteq \operatorname{int}\left(\operatorname{cl}\left(\operatorname{int}\left(F^{+}(\operatorname{scl} B)\right)\right)\right) \Rightarrow F
\end{gathered}
$$

is not f.u.a. $\alpha . c$.

Example 5.2. Let $X=\{a, b\}, \tau_{X}=\{\phi, X\}, Y=[0,1], \tau_{Y}=\left\{0_{Y}, 1_{Y}, A, B\right\}$ where $A(y)=0.4, B(y)=0.55$, for all $y \in Y$. Then $\left(X, \tau_{X}\right)$ and $\left(Y, \tau_{Y}\right)$ are a topological space and an fts respectively. Let us consider a fuzzy multifunction $F:\left(X, \tau_{X}\right) \rightarrow\left(Y, \tau_{Y}\right)$ defined by $F(a)=B, F(b)=C$ where $C(y)=0.45$, for all $y \in Y$. Here $F^{-}(A)=\phi$ and so $F^{-}(A) \subseteq \operatorname{int}\left(\operatorname{cl}\left(\operatorname{int}\left(F^{-}(\operatorname{cl} A)\right)\right)\right), F^{-}(B)=\{a\}$, $F^{-}(\operatorname{cl} B)=F^{-}\left(1_{Y} \backslash A\right)=X$ and so $F^{-}(B) \subseteq \operatorname{int}\left(\operatorname{cl}\left(\operatorname{int}\left(F^{-}(\operatorname{cl} B)\right)\right)\right) \Rightarrow F$ is

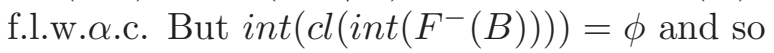

$$
F^{-}(B) \not \operatorname{int}\left(\operatorname{cl}\left(\operatorname{int}\left(F^{-}(B)\right)\right)\right) \Rightarrow F
$$

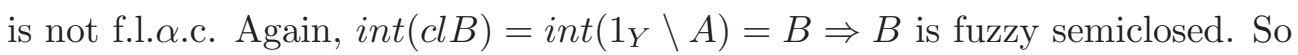

$$
\begin{array}{r}
\operatorname{int}\left(\operatorname{cl}\left(\operatorname{int}\left(F^{-}(\operatorname{scl} B)\right)\right)\right)=\operatorname{int}\left(\operatorname{cl}\left(\operatorname{int}\left(F^{-}(B)\right)\right)\right)=\phi \\
\Rightarrow F^{-}(B) \nsubseteq \operatorname{int}\left(\operatorname{cl}\left(\operatorname{int}\left(F^{-}(\operatorname{scl} B)\right)\right)\right) \Rightarrow F
\end{array}
$$

is not f.l.a. $\alpha . c$.

Example 5.3. f.u.a. $\alpha . c . ~ \Leftrightarrow$ f.u. $\alpha$.c.

Let $X=\{a, b\}, \tau_{X}=\{\phi, X\}, Y=[0,1], \tau_{Y}=\left\{0_{Y}, 1_{Y}, A, B\right\}$ where $A(y)=$ $0.5, B(y)=0.4$, for all $y \in Y$. Then $\left(X, \tau_{X}\right)$ and $\left(Y, \tau_{Y}\right)$ are a topological space and an fts respectively. Let us consider a fuzzy multifunction $F:\left(X, \tau_{X}\right) \rightarrow$ $\left(Y, \tau_{Y}\right)$ defined by $F(a)=B, F(b)=C$ where $C(y)=0.45$, for all $y \in Y$. Now $F^{+}(A)=X$ and so $F^{+}(A)=\operatorname{int}\left(\operatorname{cl}\left(\operatorname{int}\left(F^{+}(\operatorname{scl} A)\right)\right)\right)=\operatorname{int}\left(\operatorname{cl}\left(\operatorname{int}\left(F^{+}(A)\right)\right)\right)=$ $X$ (as $A$ is fuzzy semiopen in $Y$ ). $F^{+}(B)=\{a\}, F^{+}(\operatorname{scl} B)=F^{+}(A)=X$ (as the collection of all fuzzy semiopen sets in $Y$ is $\left\{0_{Y}, 1_{Y}, U\right\}$ where $B \leq U \leq A$ and so that the collection of all fuzzy semiclosed sets in $Y$ is $\left\{0_{Y}, 1_{Y}, 1_{Y} \backslash U\right\}$ where $\left.1_{Y} \backslash A \leq 1_{Y} \backslash U \leq 1_{Y} \backslash B\right)$. Therefore $F^{+}(B) \subseteq \operatorname{int}\left(\operatorname{cl}\left(\operatorname{int}\left(F^{+}(\operatorname{scl} B)\right)\right)\right)$.

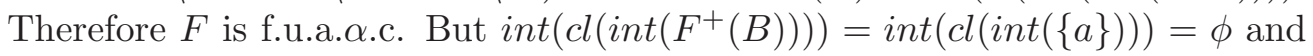

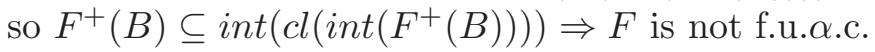

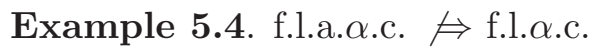


Let $X=\{a, b\}, \tau_{X}=\{\phi, X\}, Y=[0,1], \tau_{Y}=\left\{0_{Y}, 1_{Y}, B\right\}$ where $B(y)=$ 0.57 , for all $y \in Y$. Then $\left(X, \tau_{X}\right)$ and $\left(Y, \tau_{Y}\right)$ are topological space and an fts respectively. Let us consider a fuzzy multifunction $F:\left(X, \tau_{X}\right) \rightarrow\left(Y, \tau_{Y}\right)$ defined by $F(a)=B, F(b)=C$ where $C(y)=0.4$, for all $y \in Y$. Clearly $F^{-}(B)=\{a\} \Rightarrow \operatorname{int}\left(\operatorname{cl}\left(\operatorname{int}\left(F^{-}(B)\right)\right)\right)=\operatorname{int}(\operatorname{cl}(\operatorname{int}(\{a\})))=\phi \nsupseteq F^{-}(B) \Rightarrow F$ is not f.l.a.c. But the collection of all fuzzy semiopen sets in $Y$ is $\left\{0_{Y}, 1_{Y}, U\right\}$ where $U \geq B$ and that of fuzzy semiclosed sets in $Y$ is $\left\{0_{Y}, 1_{Y}, 1_{Y} \backslash U\right\}$ where $1_{Y} \backslash U \leq 1_{Y} \backslash B$. So $F^{-}(\operatorname{scl} B)=F^{-}\left(1_{Y}\right)=X$ and so $\operatorname{int}\left(\operatorname{cl}\left(\operatorname{int}\left(F^{-}(\operatorname{scl} B)\right)\right)\right)=$ $\operatorname{int}(\operatorname{cl}(\operatorname{int}(X)))=X \supseteq F^{-}(B) \Rightarrow F$ is f.l.a.Q.c.

Let us now recall a definition from [8] for ready reference.

Definition 5.5. An $\mathrm{fts}\left(Y, \tau_{Y}\right)$ is said to be fuzzy normal if for any two fuzzy closed sets $A, B$ of $Y$ with $A / q B$, there are fuzzy open sets $U, V$ in $Y$ such that $A \leq U, B \leq V$ and $U \not q V$.

Theorem 5.6. If $F: X \rightarrow Y$ is a fuzzy multifunction such that $F(x)$ is fuzzy closed in $Y$ for each $x \in X$ and $Y$ is fuzzy normal space, then the following statements are equivalent:

(i) $F$ is f.u. $\alpha . c$.

(ii) $F$ is f.u.a. $\alpha . c$.

(iii) $F$ is f.u.w.o.c.

Proof. Obviously (i) $\Rightarrow($ ii $) \Rightarrow$ (iii). So we have to prove only (iii) $\Rightarrow$ (i).

(iii) $\Rightarrow$ (i) Let $x \in X$ and $V$ be any fuzzy open set in $Y$ with $F(x) \leq V$. Then $F(x) \not q\left(1_{Y} \backslash V\right)$ where $F(x)$ and $1_{Y} \backslash V$ are fuzzy closed sets in $Y$. Since $Y$ is fuzzy normal, there exist fuzzy open sets $U, W$ in $Y$ such that $F(x) \leq U, 1_{Y} \backslash V \leq W$ and $U / q W$. Then $U \leq 1_{Y} \backslash W \Rightarrow \operatorname{cl} U \leq \operatorname{cl}\left(1_{Y} \backslash W\right)=1_{Y} \backslash W$ and so

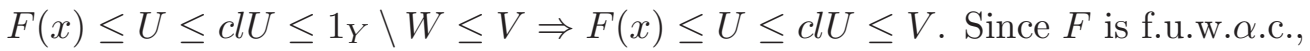

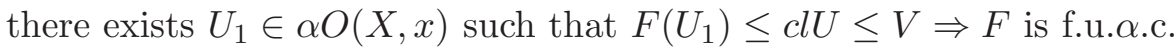

Definition 5.7. A fuzzy multifunction $F: X \rightarrow Y$ is said to be fuzzy $\alpha$-preopen if $F(U) \leq \operatorname{int}(c l(F(U)))$, for all $U \in \alpha O(X, x)$.

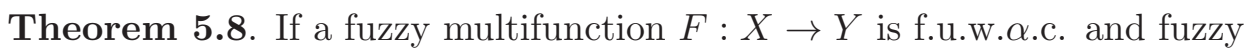
$\alpha$-preopen, then $F$ is f.u.a. $\alpha . c$.

Proof. Let $x \in X$ and $V$ be any fuzzy open set in $Y$ with $F(x) \leq V$. Since $F$ is f.u.w.Q.c., there exists $U \in \alpha O(X, x)$ such that $F(U) \leq c l V$. Since $F$ is fuzzy $\alpha$-preopen, $F(U) \leq \operatorname{int}(\operatorname{cl}(F(U))) \leq \operatorname{int}(\operatorname{cl}(c l V))=\operatorname{int}(c l V)=s c l V$, as

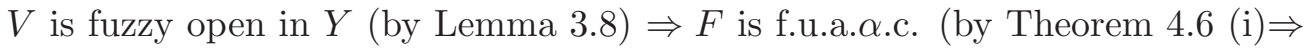
(ii)).

Theorem 5.9. Let $F: X \rightarrow Y$ be a fuzzy multifunction such that $F(x)$ is fuzzy open in $Y$ for each $x \in X$. Then the following statements are equivalent: 
(i) $F$ is f.l. $\alpha . c$.

(ii) $F$ is f.l.a. $\alpha . c$.

(iii) $F$ is f.l.w. $\alpha . c$.

Proof. Obviously (i) $\Rightarrow$ (ii) $\Rightarrow$ (iii). So we prove only (iii) $\Rightarrow$ (i).

(iii) $\Rightarrow$ (i) Let $x \in X$ and $V$ be any fuzzy open set in $Y$ with $F(x) q V$. Then by (iii), there exists $U \in \alpha O(X, x)$ such that $F(u) q c l V$, for all $u \in U$. Since by hypothesis, $F(u)$ is fuzzy open in $Y, F(u) q V$, for all $u \in U$ (if not, then $F(u) / q V$ for some $u \in U \Rightarrow F(u) / q c l V$, as $F(u)$ is fuzzy open in $Y$, a

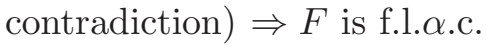

Let us now recall a definition from [9] for ready reference.

Definition 5.10. An fts $\left(Y, \tau_{Y}\right)$ is called fuzzy regular if for any fuzzy set $A$ in $Y$ and any fuzzy open set $U$ in $Y$ with $A q U$, there exists a fuzzy open set $V$ in $Y$ such that $A q V \leq c l V \leq U$.

Theorem 5.11. Let $F: X \rightarrow Y$ be f.l.w. $\alpha . c$. where $Y$ be fuzzy regular. Then $F$ is f.l.a. $\alpha$.c.

Proof. Let $x \in X$ and $V$ be fuzzy open set in $Y$ such that $F(x) q V$. Since $Y$ is fuzzy regular, there exits a fuzzy open set $U$ in $Y$ such that $F(x) q U \leq c l U \leq V$.

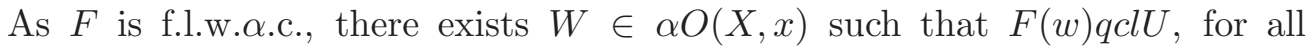
$w \in W \Rightarrow F(w) q V$, for all $w \in W \Rightarrow F(w) q s c l V$, for all $w \in W \Rightarrow F$ is f.l.a. $\alpha . c$.

We now recall the following lemma from [20].

Lemma 5.12 [20]. If a subset $A$ of a topological space $X$ is $\alpha$-open and $\alpha$-closed, then $A$ is clopen in $X$.

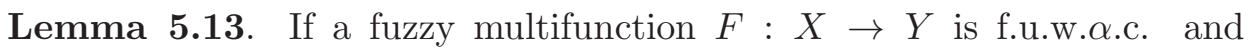

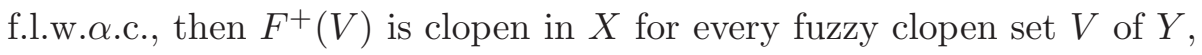

Proof. Let $V$ be fuzzy clopen in $Y$. As $F$ is f.u.w.o.c., then by Theorem $4.8(\mathrm{i}) \Rightarrow($ viii $), F^{+}(V) \subseteq \operatorname{\alpha int}\left(F^{+}(c l V)\right)=\operatorname{\alpha int}\left(F^{+}(V)\right) \Rightarrow F^{+}(V)$ is $\alpha$-open in $X$. Again, since $F$ is f.l.w. $\alpha$.c., by Theorem $4.9(\mathrm{i}) \Rightarrow(\mathrm{x}), \alpha c l\left(F^{+}(V)\right) \subseteq$ $F^{+}(c l V)=F^{+}(V) \Rightarrow F^{+}(V)$ is $\alpha$-closed in $X$. So $F^{+}(V)$ is $\alpha$-clopen in $X$. By Lemma 5.12, $F^{+}(V)$ is clopen in $X$.

\section{Applications}

We now recall the definition of connectedness of an fts from [22].

Definition 6.1. Two fuzzy sets $A$ and $B$ in an $\mathrm{fts}\left(Y, \tau_{Y}\right)$ such that $A \not q B$ and $A(x)+B(x)<1$ for $x \in A_{0} \bigcap B_{0}$ are said to be weakly separated iff 
$\operatorname{cl} A \not q B$ and $A \not q c l B .\left(Y, \tau_{Y}\right)$ is said to be fuzzy disconnected iff there are two non-zero weakly separated fuzzy sets $A$ and $B\left(A_{0} \neq B_{0}\right)$ in $\left(Y \cdot \tau_{Y}\right)$ such that $A \cup B=1_{Y}$. An fts $\left(Y, \tau_{Y}\right)$ is said to be fuzzy connected iff $Y$ is not fuzzy disconnected.

Remark 6.2. From Definition 6.1, we claim that $\left(Y, \tau_{Y}\right)$ is fuzzy disconnected if there exist two non-zero fuzzy open sets $A$ and $B$ with $A / q B$ such that $A \bigcup B=1_{Y}$ and $A \bigwedge B=0_{Y}$. Indeed, $A \bigcup B=1_{Y}$ and $A(x)+B(x)<1$, for $x \in A_{0} \bigcap B_{0} \Rightarrow A_{0} \bigcap B_{0}=\phi \Rightarrow A \wedge B=0_{Y}$ and so $A=1_{Y} \backslash B$ and so $B=1_{Y} \backslash A$. Now $\operatorname{cl} A \not q B \Rightarrow \operatorname{cl} A \leq 1_{Y} \backslash B$. Again, $\operatorname{cl} A=\operatorname{cl}\left(1_{Y} \backslash B\right)$ and so $\operatorname{cl}\left(1_{Y} \backslash B\right) \leq 1_{Y} \backslash B \Rightarrow 1_{Y} \backslash B$ is fuzzy closed in $Y$ and so $B$ is fuzzy open in $Y$. Similarly we can show that $A$ is fuzzy open in $Y$. As $B=1_{Y} \backslash A, B$ is fuzzy closed in $Y$ and similarly $A$ is fuzzy closed in $Y$.

So we can rewrite the definitions of fuzzy connectedness of a fuzzy set and an fts as follows.

Definition 6.3. A fuzzy set $A$ in an $\mathrm{fts}\left(Y, \tau_{Y}\right)$ is called disconnected if there exist two non-zero open sets $U, V$ in $Y$ such that $A=U \bigvee V$ and $U \wedge V=0_{Y}$. A is called connected if it is not disconnected.

Definition 6.4. An fts $\left(Y, \tau_{Y}\right)$ is called disconnected if there exist two non-zero open sets $U, V$ in $Y$ such that $U \bigvee V=1_{Y}$ and $U \wedge V=0_{Y} . Y$ is called connected if it is not disconnected.

Definition 6.5. A topological space is said to be disconnected if there exist two non-empty open sets $U, V$ in $X$ such that $U \cup V=X$ and $U \cap V=\phi . X$ is called connected if it is not disconnected.

Result 6.6. For a fuzzy multifunction $F: X \rightarrow Y$,

$$
F^{+}(U \bigwedge V)=F^{+}(U) \bigcap F^{+}(V)
$$

for any two fuzzy sets $U, V$ in $Y$.

Proof. Let $x \in F^{+}(U) \cap F^{+}(V)$. Then $F(x) \leq U$ and $F(x) \leq V \Rightarrow F(x) \leq$ $U \wedge V \Rightarrow x \in F^{+}(U \wedge V)$ and so $F^{+}(U) \cap F^{+}(V) \subseteq F^{+}(U \wedge V)$. Retracing the above steps, we get $F^{+}(U \wedge V) \subseteq F^{+}(U) \cap F^{+}(V)$.

Result 6.7. For a fuzzy multifunction $F: X \rightarrow Y$,

$$
F^{+}(U \bigvee V)=F^{+}(U) \bigcup F^{+}(V)
$$

for any two fuzzy sets $U, V$ in $Y$.

Proofs. Let $x \in F^{+}(U) \cup F^{+}(V)$. Then $F(x) \leq U \bigvee V \Rightarrow F(x) \leq$ $U$ or $F(x) \leq V \Rightarrow x \in F^{+}(U)$ or $x \in F^{+}(V) \Rightarrow x \in F^{+}(U) \bigcup F^{+}(V)$ 
and so $F^{+}(U \bigcup V) \subseteq F^{+}(U) \cup F^{+}(V)$. Retracing the above steps, we get $F^{+}(U) \bigcup F^{+}(V) \subseteq F^{+}(U \bigcup V)$.

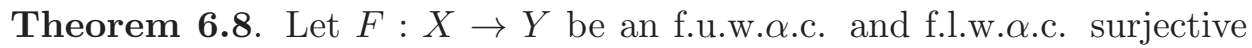
multifunction. If $X$ is connected and $F(x)$ is fuzzy connected for each $x \in X$, then $Y$ is fuzzy connected.

Proof. If possible, let $Y$ be fuzzy disconnected. Then there exist two fuzzy non-zero open sets $U, V$ in $Y$ such that $U \bigvee V=1_{Y}$ and $U \wedge V=0_{Y}$. Then $F^{+}(U \bigvee V)=F^{+}\left(1_{Y}\right)=X$ and $F^{+}(U \wedge V)=F^{+}\left(0_{Y}\right)=\phi$. Since $F(x)$ is fuzzy connected for each $x \in X$, then either $F(x) \leq U$ or $F(x) \leq V$. Then either $x \in F^{+}(U)$ or $x \in F^{+}(V) \Rightarrow x \in F^{+}(U) \cup F^{+}(V)$, for each $x \in X \Rightarrow X \subseteq$ $F^{+}(U) \cup F^{+}(V)=F^{+}(U \bigvee V)$ by Result 6.7 . Since $F$ is surjective and $U, V$ are non-zero fuzzy sets in $Y$, there exist $x, y \in X$ such that $F(x) \leq U, F(y) \leq$ $V \Rightarrow x \in F^{+}(U), y \in F^{+}(V)$ and so $F^{+}(U) \neq \phi \neq F^{+}(V)$. Therefore, we have

(i) $X=F^{+}(U \bigvee V)=F^{+}(U) \cup F^{+}(V)$

(ii) $F^{+}(U) \cap F^{+}(V)=F^{+}(U \cap V)=\phi$ (by Lemma 6.6),

(iii) $F^{+}(V) \neq \phi, F^{+}(V) \neq \phi$.

Again $U, V$ are fuzzy open in $Y$ with $U \bigvee V=1_{Y}, U \wedge V=0_{Y} \Rightarrow U=$ $1_{Y} \backslash V, V=1_{Y} \backslash U$ which shows that $U$ and $V$ are fuzzy clopen in $Y$. By Lemma $5.13, F^{+}(U)$ and $F^{+}(V)$ are clopen in $X$. Then (i), (ii) and (iii) together proves that $X$ is disconnected, a contradiction.

Definition 6.9 [20]. The $\alpha$-frontier of a subset $A$ of a topological space $X$, denoted by $\alpha \operatorname{Fr}(A)$, is defined by $\alpha \operatorname{Fr}(A)=\alpha \operatorname{cl} A \bigcap \alpha c l(X \backslash A)=\alpha c l A \backslash \alpha i n t A$.

Theorem 6.10. Let $F: X \rightarrow Y$ be a fuzzy multifunction. Let $A=\{x \in$

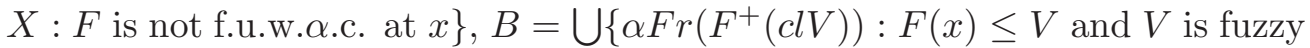
open in $Y$. Then $A=B$.

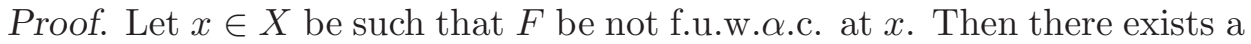
fuzzy open set $V$ in $Y$ with $F(x) \leq V$ but $U \nsubseteq F^{+}(c l V)$, for all $U \in \alpha O(X, x)$. Then $U \bigcap\left(X \backslash F^{+}(c l V)\right) \neq \phi \Rightarrow x \in \operatorname{\alpha cl}\left(X \backslash F^{+}(\operatorname{cl} V)\right)=X \backslash \operatorname{\alpha int}\left(F^{+}(c l V)\right) \Rightarrow$ $x \notin \operatorname{\alpha int}\left(F^{+}(\operatorname{cl} V)\right)$. As $x \in F^{+}(V) \subseteq F^{+}(\operatorname{cl} V) \subseteq \alpha c l\left(F^{+}(\operatorname{cl} V)\right) \Rightarrow x \in$ $\alpha F r\left(F^{+}(c l V)\right) \Rightarrow A \leq B$.

Conversely, let $x \in X$ and $V$ be fuzzy open set in $Y$ with $F(x) \leq V$ such

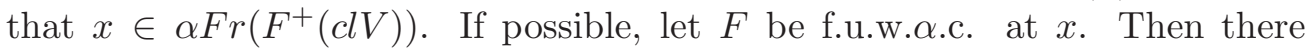
exists $U \in \alpha O(X, x)$ such that $U \subseteq F^{+}(c l V)$. Then $x \in U=\operatorname{\alpha int} U \subseteq$ $\operatorname{\alpha int}\left(F^{+}(c l V)\right) \Rightarrow x \in \operatorname{\alpha int}\left(F^{+}(c l V)\right) \Rightarrow x \notin \alpha F r\left(F^{+}(c l V)\right)$, a contradiction

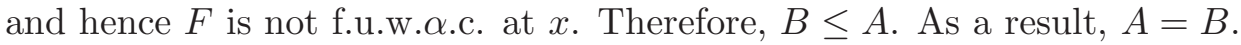

Theorem 6.11. Let $F: X \rightarrow Y$ be a fuzzy multifunction. Let $A=\{x \in$ $X: F$ is not f.l.w. $\alpha$.c. at $x\}, B=\bigcup\left\{\alpha F r\left(F^{-}(c l V)\right): F(x) q V\right.$ and $V$ is fuzzy open in $Y$. Then $A=B$. 
Proof. Let $x \in X$ be such that $F$ be not f.l.w. $\alpha . c$. at $x$. Then there exists a fuzzy open set $V$ in $Y$ with $F(x) q V$ but $U \nsubseteq F^{-}(c l V)$, for all $U \in \alpha O(X, x)$. Then $U \bigcap\left(X \backslash F^{-}(c l V)\right) \neq \phi \Rightarrow x \in \alpha c l\left(X \backslash F^{-}(c l V)\right)=X \backslash \operatorname{\alpha int}\left(F^{-}(c l V)\right) \Rightarrow$ $x \notin \operatorname{\alpha int}\left(F^{-}(\operatorname{cl} V)\right)$. As $x \in F^{-}(V) \subseteq F^{-}(\operatorname{cl} V) \subseteq \alpha c l\left(F^{-}(c l V)\right) \Rightarrow x \in$ $\alpha \operatorname{Fr}\left(F^{-}(c l V)\right) \Rightarrow A \leq B$.

Conversely, let $x \in X$ and $V$ be fuzzy open set in $Y$ with $F(x) q V$ such that $x \in \alpha \operatorname{Fr}\left(F^{-}(c l V)\right)$. If possible, let $F$ be f.l.w. $\alpha . c$. at $x$. Then there exists $U \in \alpha O(X, x)$ such that $U \subseteq F^{-}(c l V)$. Then $x \in U=\alpha i n t U \subseteq$ $\operatorname{\alpha int}\left(F^{-}(c l V)\right) \Rightarrow x \in \operatorname{\alpha int}\left(F^{-}(c l V)\right) \Rightarrow x \notin \alpha F r\left(F^{-}(c l V)\right)$, a contradiction and hence $F$ is not f.l.w. $\alpha . c$. at $x$. Therefore, $B \leq A$. As a result, $A=B$.

Definition 6.12. A fuzzy set $A$ in an fts $Y$ is said to be a fuzzy upper (lower) $C$-nbd of a fuzzy set $B$ in $Y$ if there exists a fuzzy open set $V$ in $Y$ such that $B q V$ (resp., $B \leq V)$ and $c l V \not q\left(1_{Y} \backslash A\right)$.

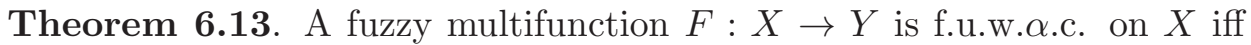
for each point $x_{0} \in X$ and each fuzzy upper (lower) $C$-nbd $M$ of $F\left(x_{0}\right), F^{+}(M)$ (resp., $\left.F^{-}(M)\right)$ is an $\alpha$-nbd of $x_{0}$.

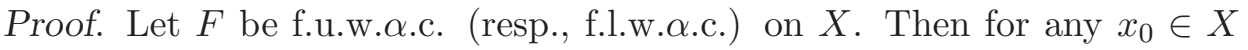
and for any fuzzy upper (lower) $C$-nbd $M$ of $F\left(x_{0}\right)$, there exists a fuzzy open set $V$ in $Y$ such that $F\left(x_{0}\right) \leq V$ (resp., $\left.F\left(x_{0}\right) q V\right)$ and $c l V \not q\left(1_{Y} \backslash M\right) \Rightarrow c l V \leq M$.

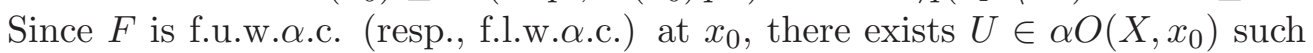
that $U \subseteq F^{+}(c l V)$ (resp., $\left.U \subseteq F^{-}(c l V)\right) \subseteq F^{+}(M)$ (resp., $U \subseteq F^{-}(M)$ ) $\Rightarrow F^{+}(M)$ (resp., $\left.F^{-}(M)\right)$ is an $\alpha$-nbd of $x_{0}$.

Conversely, let for any $x_{0} \in X$ and any fuzzy open set $V$ of $Y$ with $F\left(x_{0}\right) \leq$ $V$ (resp., $F\left(x_{0}\right) q V$ ), we have $c l V \not q\left(1_{Y} \backslash c l V\right) \Rightarrow c l V$ is a fuzzy upper (lower) $C$-nbd of $F\left(x_{0}\right)$. Then by hypothesis, $F^{+}(c l V)$ (resp., $F^{-}(c l V)$ ) is an $\alpha$-nbd of $x_{0} \Rightarrow$ there exists $U \in \alpha O\left(X, x_{0}\right)$ such that $x_{0} \in U \subseteq F^{+}(c l V)$ (resp.,

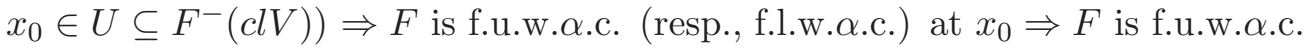

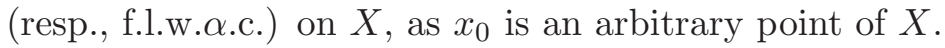

Definition 6.14 [11]. A topological space $X$ is said to be $\alpha$-compact if every cover of $X$ by $\alpha$-open sets of $X$ has a finite subcover.

Definition 6.15 [7]. A fuzzy set $A$ in an $\mathrm{fts}\left(Y, \tau_{Y}\right)$ is said to be fuzzy compact set if every fuzzy open cover of $A$ has a finite subcover for $A$.

Definition 6.16. An fts $\left(Y, \tau_{Y}\right)$ is called fuzzy $s^{*}$-compact if every fuzzy open cover $\mathcal{U}$ of $Y$ has a finite $s$-proximate subcover (i.e., there exists a finite subcollection $\mathcal{U}_{0}$ of $\mathcal{U}$ such that $\left\{s c l U: U \in \mathcal{U}_{0}\right\}$ covers $Y$.

Theorem 6.17. Let $F: X \rightarrow Y$ be an f.u.w. $\alpha . c$. surjective multifunction and $F(x)$ be a fuzzy compact set in $Y$ for each $x \in X$. If $X$ is $\alpha$-compact, then 
$Y$ is fuzzy almost compact.

Proof. Let $\mathcal{A}=\left\{A_{\alpha}: \alpha \in \Lambda\right\}$ be a fuzzy open cover of $Y$. Now for each $x \in X, F(x)$ is fuzzy compact in $Y$ and so there is a finite subset $\Lambda_{x}$ of $\Lambda$ such that $F(x) \leq \bigcup\left\{A_{\alpha}: \alpha \in \Lambda_{x}\right\}$. Let $A_{x}=\bigcup\left\{A_{\alpha}: \alpha \in \Lambda_{x}\right\}$. Then $A_{x}$ is fuzzy open in $Y$ such that $F(x) \leq A_{x}$. Since $F$ is f.u.w. $\alpha$.c. on $X$, there exists $U_{x} \in \alpha O(X, x)$ such that $U_{x} \subseteq F^{+}\left(c l A_{x}\right)$. Then $\mathcal{U}=\left\{U_{x}: x \in X\right\}$ is a cover of $X$ by $\alpha$-open sets of $X$. As $X$ is $\alpha$-compact, there are finitely many points $x_{1}, x_{2}, \ldots, x_{n}$ in $X$ such that $X=\bigcup_{i=1}^{n} U_{x_{i}}$. As $F$ is surjective, $1_{Y}=F(X)=$ $F\left(\bigcup_{i=1}^{n} U_{x_{i}}\right)=\bigcup_{i=1}^{n} F\left(U_{x_{i}}\right) \leq \bigcup_{i=1}^{n} \operatorname{cl} A_{x_{i}}=\bigcup_{i=1}^{n} \operatorname{cl}\left(\bigcup_{\alpha \in \Lambda_{x_{i}}} A_{\alpha}\right)=\bigcup_{i=1}^{n}\left(\bigcup_{\alpha \in \Lambda_{x_{i}}} c l A_{\alpha}\right) \Rightarrow Y$ is fuzzy almost compact.

Now we state the following two theorems, the proofs of these two are analogue to the proof of Theorem 6.17.

Theorem 6.18. Let $F: X \rightarrow Y$ be an f.u.a. $\alpha . c$. surjective multifunction and $F(x)$ be a fuzzy compact set in $Y$ for each $x \in X$. If $X$ is $\alpha$-compact, then $Y$ is fuzzy $s^{*}$-compact.

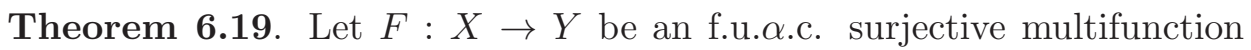
and $F(x)$ be a fuzzy compact set in $Y$ for each $x \in X$. If $X$ is $\alpha$-compact, then $Y$ is fuzzy compact.

\section{References}

[1] D. Andrijević, Some properties of the topology of $\alpha$-sets, Mat. Vesnik, 36 (1984), 1-10.

[2] K.K. Azad, On fuzzy semi-continuity, fuzzy almost continuity and fuzzy weakly continuity, J.Math. Anal. Appl., 82 (1981), 14-32.

[3] Anjana Bhattacharyya, Concerning almost quasi continuous fuzzy multifunctions, Universitatea Din Bacău Studii Şi Cercetări Ştiințifice, Seria: Matematică, Nr. 11 (2001), $35-48$.

[4] Anjana Bhattacharyya, Fuzzy $\gamma$-continuous multifunction, Int. Journal of Advanced Research in Science and Engineering, 4 Issue No. 02 (2015), 195-209.

[5] A.S. Bin Shahna, On fuzzy strong semicontinuity and fuzzy precontinuity, Fuzzy Sets and Systems, 44 (1991), 303-308.

[6] C.L. Chang, Fuzzy topological spaces, J. Math. Anal. Appl., 24 (1968), 182-190.

[7] S. Ganguly, S. Saha, A note on compactness in fuzzy setting, Fuzzy Sets and Systems, 34 (1990), 117-124.

[8] B. Hutton, Normality in fuzzy topological spaces, J. Math. Anal. Appl., 50 (1975), 74-79. 
[9] B. Hutton, I. Reilly, Separation axioms in fuzzy topological spaces, Fuzzy Sets and Systems, 31 (1980), 93-104.

[10] N. Levine, Semi-open sets and semi-continuity in topological spaces, Amer. Math. Monthly, 70 (1963), 36-41.

[11] S.N. Maheshwari, S.S. Thakur, On $\alpha$-compact spaces, Bull. Inst. Math. Acad. Sinica, 13 (1985), 341-347.

[12] A.S. Mashhour, M.E. Abd El-Monsef, S.N. El-Deeb, On precontinuous and weak precontinuous mappings, Proc. Math. Phys. Soc. Egypt, 53 (1982), 47-53.

[13] A.S. Mashhour, I.A. Hasanein, S.N. El-Deeb, $\alpha$-continuous and $\alpha$-open mappings, Acta Math. Hungar., 41 (1983), 213-218.

[14] M.N. Mukherjee, S. Malakar, On almost continuous and weakly continuous fuzzy multifunctions, Fuzzy Sets and Systems, 41 (1991), 113-125.

[15] M.N. Mukherjee, S.P. Sinha, Almost compact fuzzy sets in fuzzy topological spaces, Fuzzy Sets and Systems, 38 (1990), 389-396.

[16] M.N. Mukherjee, S.P. Sinha, Fuzzy $\theta$-closure operator on fuzzy topological spaces, Internat. J. Math. and Math. Sci., 14, No. 2 (1991), 309-314.

[17] T. Noiri, On semi-continuous mappings, Atti Acad. Naz. Lincei. Rend. Cl. Sci. Fis. Mat. Natur. 54, No. 8 (1973), 210-214.

[18] T. Noiri, A note on semi-continuous mappings, Atti Acad. Naz. Lincei Rend. Cl. Sci. Fis. Mat. Natur. 55, No. 8 (1973), 400-403.

[19] N.S. Papageorgiou, Fuzzy topology and fuzzy multifunctions, Jour. Math. Anal. Appl., 109 (1985), 397-425.

[20] V. Popa, T. Noiri, On upper and lower weakly $\alpha$-continuous multifunctions, Novi. Sad. J. Math., 32, No. 1 (2002), 7-24.

[21] Pao Ming Pu, Ying Ming Liu, Fuzzy topology I. Neighbourhood structure of a fuzzy point and Moore-Smith convergence, Jour. Math. Anal. Appl., 76 (1980), 571-599.

[22] S. Saha, Local connectedness in a fuzzy setting, Simon Stevin, 61 (1987), 3-13.

[23] L.A. Zadeh, Fuzzy Sets, Inform. Control, 8 (1965), 338-353. 